\title{
Transactional Associations Between Parent and Late Adolescent Internalizing Symptoms During the COVID-19 Pandemic: The Moderating Role of Avoidant Coping
}

\author{
Nicole E. Lorenzo $\mathbb{1}^{1}$ - Selin Zeytinoglu ${ }^{1}$ - Santiago Morales ${ }^{1} \cdot$ Jamie Listokin $^{1} \cdot$ Alisa N. Almas ${ }^{2}$. \\ Kathryn A. Degnan ${ }^{3} \cdot$ Heather Henderson ${ }^{4} \cdot$ Andrea Chronis-Tuscano $^{1} \cdot$ Nathan A. Fox $^{1}$
}

Received: 30 September 2020 / Accepted: 4 December 2020 / Published online: 25 January 2021

(c) The Author(s), under exclusive licence to Springer Science+Business Media, LLC part of Springer Nature 2021

\begin{abstract}
Extensive research has demonstrated the transactional nature of parent-child psychopathology, with limited studies examining these effects during late adolescence and none, to our knowledge, longitudinally during the COVID-19 pandemic. The current study examined the cross-lagged effects of parent and adolescent internalizing symptoms during the COVID-19 pandemic and the moderating role of avoidant coping. A sample of 291 adolescents (Age mean $=18.27 ; 53 \%$ female; 61\% White) and their parents rated their own anxiety and depressive symptoms and coping during the first two months following stay-at-home orders during the COVID-19 pandemic. Parent internalizing symptoms at the first assessment predicted adolescent internalizing symptoms at the second assessment. Adolescent avoidant coping style moderated this effect of parent internalizing symptoms on adolescent internalizing symptoms in the subsequent month, such that parent internalizing symptoms predicted child internalizing symptoms only among adolescents with moderate to high rates of avoidant coping. Follow-up analyses indicated different patterns when examining depressive and anxiety symptoms separately. The results highlight complex family dynamics between adolescents and their parents and begin to differentiate how individual characteristics impact the response to a significant life event such as the COVID-19 pandemic.
\end{abstract}

Keywords COVID-19 $\cdot$ Internalizing symptoms $\cdot$ Adolescents $\cdot$ Parents $\cdot$ Coping

\section{Introduction}

The COVID-19 pandemic placed a significant strain on families, creating important challenges to mental health, such as increased rates of anxiety and depression in parents and their children (Cameron et al. 2020; Park et al. 2020). Late adolescence may be a particularly difficult time for

Nicole E. Lorenzo

nlorenzo@umd.edu

1 University of Maryland, 3942 Campus Drive, College Park, MD 20742, USA

2 University of British Columbia, 2206 East Mall, Vancouver, BC V6T 1Z3, Canada

3 Catholic University, 620 Michigan Ave., N.E., Washington, DC 20064, USA

4 Waterloo University, 200 University Avenue West, Waterloo, ON N2L 3G1, Canada individuals to experience the changes associated with the COVID-19 pandemic. Specifically, late adolescence is a critical developmental period during which adolescents become increasingly independent. However, the restrictions in place during the first few months of the COVID-19 pandemic (e.g., physical distancing, stay-at-home orders, quarantine, school closures) limited the independence of many late adolescents, meanwhile increasing their proximity to family. These changes may meaningfully impact late adolescents and their parents, heightening risk for symptoms of anxiety and depression within these families. As such, identifying the transactional associations between parents' and late adolescents' anxiety and depressive symptoms, as well as individual risk factors that amplify internalizing symptoms, is crucial during times of restrictions that significantly impact their daily lives. The goals of the current study are to (a) examine the cross-lagged relations between parent internalizing symptoms and adolescent internalizing symptoms over time during two early months of the COVID pandemic, (b) examine whether adolescent 
and parent avoidant coping moderates these relations, and (c) explore these models for anxiety and depressive symptoms separately.

Previous research has shown an association between parental anxiety and depression and child anxiety and depression, with higher rates of parental depression (Goodman and Brand 2011) and anxiety (Pereira et al., 2014) related to higher levels of child internalizing symptoms, broadly speaking. With regard to unprecedented life events, such as the Boston Marathon bombing, child internalizing symptoms were worse among children of highly distressed caregivers (Kerns et al. 2014). Further, a developmental transactional model of parent-child psychopathology holds that parents and children exert dynamic, reciprocal influences on one another (Ciciolla et al., 2014). Parents' anxiety and depressive symptoms may impact children's ability to cope with negative emotions and stressors, and ultimately the course of children's psychopathology, as well as the inverse (Morris et al. 2007). Research in Spain and Italy provided initial evidence that parents reporting higher levels of stress due to the COVID19 quarantine also reported that their children experienced higher levels of internalizing symptoms (Orgiles et al. 2020). However, this work was cross-sectional, included school-age children, and only included parent report, limiting the conclusions about directionality. Moreover, not all families were affected in the same way by the pandemic and some are inherently more prone to experiencing increased internalizing symptoms. As such, it is important to identify which families are at greatest risk for increased internalizing symptoms during unprecedented situations that involve abrupt changes in developmental contexts, such as increased contact between parents and their children (e.g., stay-at-home orders). To date, there is limited knowledge on the secondary effects of the COVID-19 pandemic on the well-being of the family system, particularly with late adolescents and their parents, and the individual risk factors that moderate these relations.

One potential moderator of the transactional impact of parent and adolescent internalizing symptoms is the way in which parents and adolescents cope with stress. Research has shown that during stressful situations, some individuals engage in potentially maladaptive or avoidant coping strategies such as distraction, behavioral disengagement, selfblame, or substance use (Park et al. 2020). During the COVID-19 pandemic, research has shown increased rates of withdrawal and media use as a form of avoidant coping (Pahayahay and Khalili-Mahani 2020). Avoidant coping skills have been shown to exacerbate trauma-related stress in adults (Ehring et al. 2011) and adolescents (Ollendick et al., 2001) and place adolescents and parents at greater risk of increasing levels of mental health symptoms, including anxiety (Wilkinson et al. 2000) and depression
(Seiffge-Krenke and Klessinger 2000). As such, greater use of avoidant coping by late adolescents and their parents may increase their susceptibility to the other's anxiety and depressive symptoms.

\section{Current Study}

Using a cross-lagged, longitudinal moderation model, the current study sought to examine the transactional effects of parent and late adolescents' internalizing symptoms, and the moderating effect of avoidant coping on these relations, across the first two months of stay-at-home orders, during the COVID-19 pandemic in the US state of Maryland. It was hypothesized that higher levels of parent internalizing symptoms at Time 1 (i.e., approximately one-month after stay-at-home orders) would predict increased adolescent internalizing symptoms at Time 2 (i.e., approximately twomonths after stay-at-home orders), and that higher levels of adolescent internalizing symptoms at Time 1 would predict increased parent internalizing symptoms at Time 2. Given the associations of avoidant coping with anxiety and depressive symptoms, it was also expected that higher levels of adolescent avoidant coping would moderate the effect of parent internalizing symptoms on late adolescent internalizing symptoms. Similarly, it was expected that higher levels of parent avoidant coping would moderate the effect of late adolescent internalizing symptoms on parent internalizing symptoms. Exploratory analyses also examined these models for anxiety and depressive symptoms separately.

\section{Methods}

\section{Participants}

Participants in the current study were part of a larger, longitudinal study, conducted in a large metropolitan city in the Mid-Atlantic region of the United States, designed to examine links between temperament and psychopathology. Participants were 291 children (135 male, 156 female) selected at 4 months of age based on temperamental reactivity and their parents. For additional details on the recruitment and screening for the longitudinal sample see (Hane et al. 2008). Based on parent report at recruitment, maternal demographics included $69.1 \%$ White, $16.5 \%$ Black, $7.2 \%$ Hispanic, 3.1\% Asian, 3.4\% other, and $0.7 \%$ missing; paternal demographics included $68.7 \%$ White, $18.6 \%$ Black, $5.5 \%$ Hispanic, $2.7 \%$ Asian, $3.1 \%$ other, and $1.4 \%$ missing. At recruitment, most of the mothers in the sample had a college degree or higher $(77.6 \%)$, some had a high school degree $(16.2 \%)$, and a few reported other 
education $(5.5 \%)$. At recruitment, most of the fathers in the sample had a college degree or higher $(71.9 \%)$, some had a high school degree (19.6\%), and a few reported other education $(6.9 \%)$. Based on clinical diagnostic interviews conducted when adolescents were 15 and 18 years old, 21 (13\%) met criteria for generalized anxiety disorder and 10 $(6 \%)$ met criteria for depression.

For purposes of the current study, adolescents $(M=$ 18.27, $S D=0.665)$, and their parents completed questionnaires online via REDCap (i.e., online survey application) between April 20th and May 15th of 2020, approximately one month $(M=29.67$ days, $S D=$ 6.01 days) after a stay-at-home order was implemented in Maryland on March 30 (Time 1). Approximately one month later $(M=26.48$ days, $S D=7.31$ days $)$, participants completed a second assessment (Time 2) after the mandatory stay-at-home order was lifted and restrictions were slowly reduced, in the state of Maryland. Specifically, 156 adolescent participants and 173 parent participants $(90 \%$ mothers) completed the first assessment during the pandemic. Of the participants who completed the assessments for the current study compared to the original sample, more adolescent participants were female $(n=91,58.7 \%)$; maternal demographics included $76 \%$ White, $11 \%$ Black, 5\% Hispanic, 3\% Asian, 3\% other, and 0.6\% missing; paternal demographics included $80 \%$ White and $20 \%$ Hispanic. The Institutional Review Board at the University of Maryland approved all study protocols as meeting ethical guidelines and all participants were compensated for their time.

\section{Measures}

\section{Avoidant coping}

Avoidant coping was measured using the avoidant coping scale of the Brief COPE Inventory at two assessments during the COVID-19 pandemic, self-reported by parents and adolescents. The Brief COPE is a 28-item self-report questionnaire designed to assess use of coping strategies during stressful life events (Carver 1997). The avoidant coping scale (10 items) is the average of the denial, substance use, behavioral disengagement, self-distraction, and self-blame subscales, each of which included two items. Items ranged from a scale of 1, "I haven't been doing this at all," to 4, "I've been doing this a lot," (Baumstarck et al. 2017). Higher scores indicated a higher tendency to engage in avoidant coping in response to stressful life events. The Brief COPE has been shown to have good internal reliability (Yusoff, 2011), and good convergent and discriminant validity (Carver 1997). The avoidant scale showed questionable internal reliability in the current study for parent reports, $\alpha=0.63$, with limited variability
$(S D=0.029)$, and good internal reliability for adolescent participants, $\alpha=0.75$.

\section{Anxiety symptoms}

Adolescents and parents completed the Generalized Anxiety Disorder-7 (GAD-7; Spitzer et al. 2006) and the Patient Health Questionnaire-8 (PHQ-8; Kroenke et al. 2009) at two assessments during the COVID-19 pandemic. GAD-7 items consisted of various anxiety symptoms, rated on a four-point scale (i.e., 0-not at all to 3-nearly every day), and were summed to create an overall score. Higher scores indicated greater anxiety and a score $\geq 10$ is considered to be in the clinical range. This scale has been shown to have high test-retest reliability and good convergent validity (Spitzer et al. 2006). The scale showed excellent internal consistency for adolescent participants at both time points, $\alpha$ 's $>0.92$ and good test re-test reliability, $r=0.81$, as well as for parent participants; good internal consistency, $\alpha$ 's $>0.85$, and test re-test reliability, $r=0.72$. Higher scores indicated greater anxiety symptoms.

\section{Depressive symptoms}

The PHQ-8 is a short form version of the Patient Health Questionnaire created to assess symptoms of depression (Kroenke et al. 2009). It consists of 8 items that are scored using a 4-point Likert Scale from 0 (not at all) to 3 (nearly every day). Both internal and test-retest reliability of the PHQ-8 are strong (Kroenke et al. 2009). Higher scores on the PHQ-8 indicate increased risk for a diagnosis of various depressive disorders, with scores $\geq 10$ considered in the clinical range. The scale showed good internal consistency for adolescent participants at both time points, $\alpha$ 's $>0.88$, and good test re-test reliability, $r=$ 0.71 , as well as parent participants: $\alpha$ 's $>0.82$, and test retest reliability, $r=0.78$. Anxiety (GAD-7) and depressive (PHQ-8) symptom scores were summed to create an internalizing problem score, as in previous studies (Kroenke et al. 2016). Higher scores indicated greater internalizing problems.

\section{Data Analysis}

To examine the aims of the present study, a cross-lagged moderation path model was conducted using Mplus 8.0 (Muthén and Muthén 1998-2017). See Fig. 1 for the path model. Given the high correlation between anxiety and depressive symptoms at Time 1 for adolescent, $r=0.704$, $p<0.001$, and parent, $r=0.700, p<0.001$, participants, as well as support for combining the two constructs in the literature (Kroenke et al. 2019), internalizing composites were created for adolescent and parent participants by 


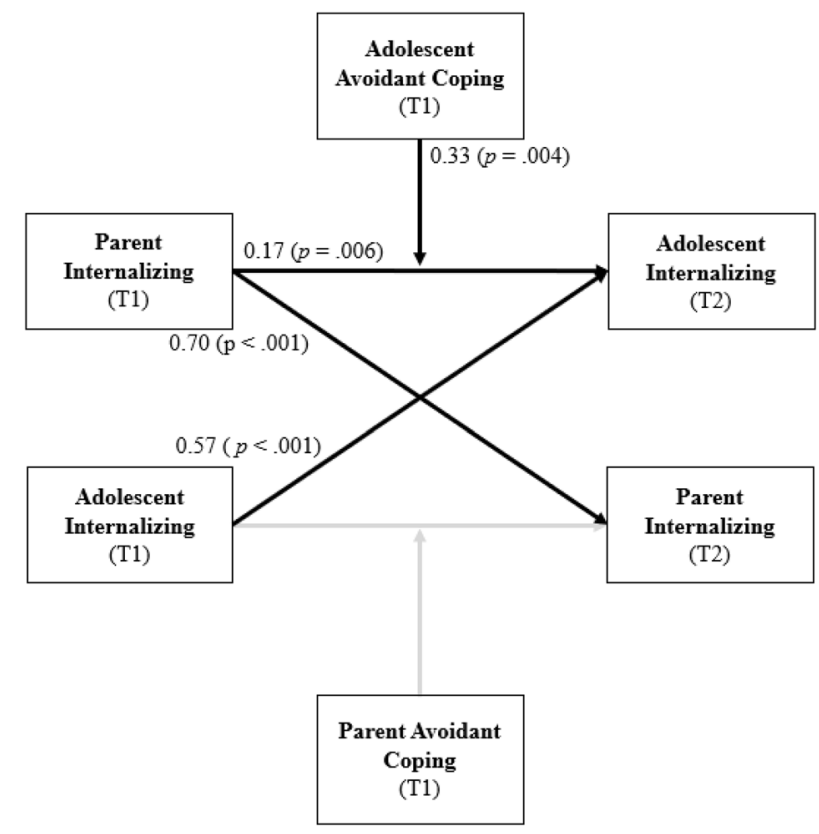

Fig. 1 Path diagram illustrating the moderated model tested. Covariates included in the analyses are not displayed in the model for visual purposes. Bolded paths represent significant paths with unstandardized estimates. T1 and T2 represent the first and second assessments, approximately one and two-months after stay-at-home orders during the COVID-19 pandemic

summing GAD and PHQ scores (Kroenke et al. 2016). To test the moderating effect of adolescent avoidant coping on the relation between parent internalizing symptoms and adolescent internalizing symptoms, parent internalizing symptoms and adolescent avoidant coping were meancentered, and a product interaction term was created. Additionally, to test the moderating effect of parent avoidant coping in the relation between adolescent internalizing symptoms and parent internalizing symptoms, adolescent internalizing symptoms and parent avoidant coping were mean-centered, and a product term was created.

\section{Sensitivity Analyses}

Given the potential clinical and intervention implications that may be different for anxiety and depressive symptoms, two additional cross-lagged models were tested as exploratory analyses: one for anxiety symptoms and one for depressive symptoms. Mean centering was used before creating interaction terms between parent anxiety symptoms and adolescent avoidant coping, adolescent anxiety symptoms and parent avoidant coping, parent depressive symptoms and adolescent avoidant coping, and adolescent depressive symptoms and parent avoidant coping. Although avoidant coping was the expected coping moderator, for sensitivity analyses the other coping scales (i.e., positive thinking, social support, problem solving) were explored.
The results for all other scales demonstrated non-significant moderation effects $(p=0.29-0.95)$ and therefore are not presented.

\section{Missing Data and Attrition}

In order to test whether participants who provided data during the COVID-19 assessments differed from the participants in the original sample of infants, chi-square analyses comparing these two samples in relation to child gender, and mothers' ethnicity and education were conducted. Mother's ethnicity (non-Hispanic, White vs. other) was associated with missing data at Time $2, \chi^{2}(1)=5.58, p=$ 0.018 , such that participants with available data were more likely to have non-Hispanic, White mothers. Therefore, maternal ethnicity was included as a covariate in the analyses, Non-Hispanic, White $=1$ and Other $=0$. Missing data on all other variables was not significantly associated with any demographic variables or variables included in the model, $p$ 's $>0.05$. In addition, adolescent sex, adolescent mean age across both assessments, the time between assessments during the pandemic for both parents and adolescents, and the days between the assessment and the stay-at-home order for both parents and adolescents, were included as covariates (See Table 1 for more descriptive information).

Attrition between Time 1 and Time 2 was minimal. Specifically, for adolescent participants, nine participants did not complete Time 2, and for parents, only seven participants. Additionally, seven adolescent participants and nine parent participants completed Time 2, but not Time 1 . In order to correct for any departures from multivariate normality, the model was estimated using maximum likelihood with robust standard errors (MLR). Missing data was handled using full information maximum likelihood estimation (FIML), which has been shown to reduce bias in parameter estimates while maintaining the full sample of participants $(N=291$; Enders and Bandalos 2001). Of note, an additional model using only participants who completed the assessments for the current study yielded the same pattern of results.

\section{Results}

\section{Internalizing Symptoms during the COVID-19 Pandemic}

Descriptive statistics and the correlations among all study variables are presented in Table 1. At Time 1 (T1), $21 \%$ of adolescent participants reported clinically significant levels of anxiety symptoms (i.e., scores $\geq 10$ ) and $30 \%$ reported clinically significant levels of depressive symptoms 


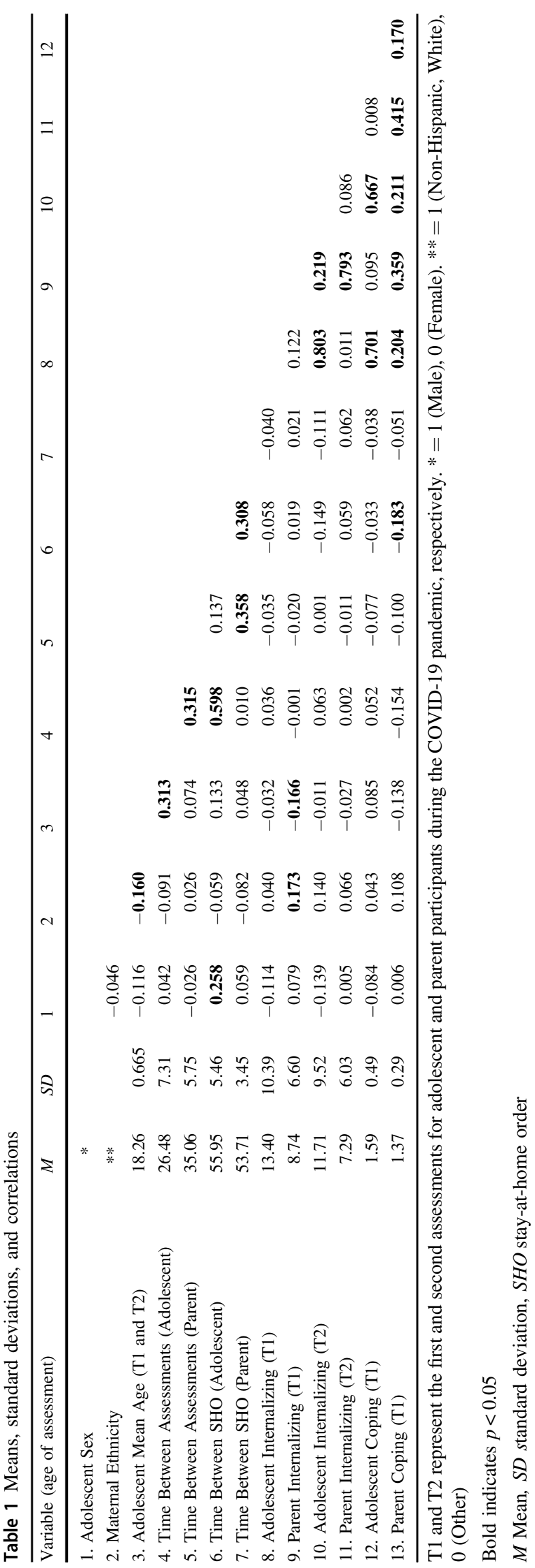

(i.e., scores $\geq 10$ ). Of the parent participants, $8 \%$ reported clinically significant levels of anxiety symptoms and $9 \%$ reported clinically significant levels of depressive symptoms. At Time 2 (T2), 18\% of adolescent participants reported clinically significant levels of anxiety symptoms and $25 \%$ reported clinically significant levels of depressive symptoms. Of the parent participants, 6\% reported clinically significant levels of anxiety symptoms and $8 \%$ reported clinically significant levels of depressive symptoms. The results from paired samples t-tests indicated a significant decrease in adolescent internalizing symptoms, $t(145)=3.26, p=0.001$, from $\mathrm{T} 1$ to $\mathrm{T} 2$, and parent internalizing symptoms, $t(162)=4.36, p<0.001$, from T1 to T2. Parent internalizing symptoms and adolescent internalizing symptoms were not correlated at T1, $p=0.094$, or $\mathrm{T} 2, p=0.767$.

\section{Cross-Lagged Moderation Model of Adolescent and Parent Internalizing Symptoms and Avoidant Coping}

The cross-lagged moderation model fit the data well, $\mathrm{RMSEA}=0.00 \quad[90 \% \quad \mathrm{CI}=0.00-0.23] ; \quad \mathrm{CFI}=1.00$; $\mathrm{SRMR}=0.024$. All unstandardized and standardized path coefficients, including the covariates, are reported in Table 2. As expected, there was significant stability in T1 and $\mathrm{T} 2$ parent and adolescent internalizing symptoms. There was also a direct effect of $\mathrm{T} 1$ parent avoidant coping on $\mathrm{T} 2$ parent internalizing, and $\mathrm{T} 1$ adolescent avoidant coping on T2 adolescent internalizing. However, crosslagged effects between parent and adolescent participants were only significant for $\mathrm{T} 1$ parent internalizing on $\mathrm{T} 2$ adolescent internalizing, and not the inverse.

Additionally, when examining the moderating effect of coping, the results showed that the relation between $\mathrm{T} 1$ parent internalizing symptoms and $\mathrm{T} 2$ adolescent internalizing was moderated by $\mathrm{T} 1$ adolescent avoidant coping, $b=0.33, S E=0.11, p=0.004$. As shown in Fig. 2, higher parent internalizing at $\mathrm{T} 1$ predicted higher adolescent internalizing symptoms at T2 when adolescent avoidant coping was moderate (i.e., mean level), $b=0.17$, $S E=0.06, p=0.006$, and high (i.e., $+1 \mathrm{SD}$ ), $b=0.34$, $S E=0.09, p<0.001$, but not when adolescent avoidant coping was low (i.e., $-1 \mathrm{SD}$ ), $b=0.01, S E=0.07, p=$ 0.914. Further analyses, using confidence intervals suggests that avoidant coping moderates the relation between parent internalizing symptoms and adolescent internalizing symptoms, such that the association is significant at 0.50 standard deviations below the mean and higher. There was no significant interaction between T1 adolescent internalizing symptoms and $\mathrm{T} 1$ parent avoidant coping on T2 parent internalizing symptoms, $b=0.13$ $S E=0.12, p=0.274$. 
Table 2 Standardized and unstandardized estimates for dual moderation model

\begin{tabular}{|c|c|c|c|c|c|c|c|}
\hline Outcome & Predictors & $\beta$ & $B$ & SE & $p$ & CI Lower & CI Upper \\
\hline Adolescent Internalizing (T2) & Adolescent Internalizing (T1) & 0.57 & 0.61 & 0.07 & 0.000 & 0.432 & 0.710 \\
\hline Adolescent Internalizing (T2) & Parent Internalizing (T1) & 0.17 & 0.11 & 0.04 & 0.006 & 0.048 & 0.290 \\
\hline Adolescent Internalizing (T2) & Adolescent Avoidant Coping (T1) & 3.69 & 0.18 & 0.07 & 0.007 & 0.986 & 6.391 \\
\hline Adolescent Internalizing (T2) & $\begin{array}{l}\text { Interaction (Parent Internalizing and Adolescent } \\
\text { Coping) }\end{array}$ & 0.33 & 0.13 & 0.05 & 0.004 & 0.104 & 0.559 \\
\hline Adolescent Internalizing (T2) & Adolescent Sex & -0.78 & -0.04 & 0.05 & 0.387 & -2.554 & 0.990 \\
\hline Adolescent Internalizing (T2) & Adolescent Mean Age & 0.07 & -0.01 & 0.05 & 0.919 & -1.248 & 1.384 \\
\hline Adolescent Internalizing (T2) & Maternal Ethnicity & 1.43 & 0.07 & 0.05 & 0.133 & -0.435 & 3.292 \\
\hline Adolescent Internalizing (T2) & Time Between Stay-at-Home Order (Adolescent) & $-\mathbf{0 . 3 2}$ & $-\mathbf{0 . 1 8}$ & 0.06 & 0.002 & $-\mathbf{0 . 5 3 1}$ & -0.117 \\
\hline Adolescent Internalizing (T2) & Time Between Assessments (Adolescent) & 0.20 & 0.15 & 0.06 & 0.008 & 0.052 & 0.356 \\
\hline Parent Internalizing (T2) & Parent Internalizing (T1) & 0.70 & 0.76 & 0.05 & 0.000 & 0.584 & 0.820 \\
\hline Parent Internalizing (T2) & Adolescent Internalizing (T1) & -0.06 & -0.10 & 0.05 & 0.052 & -0.116 & 0.001 \\
\hline Parent Internalizing (T2) & Parent Avoidant Coping (T1) & 3.04 & 0.15 & 0.07 & 0.026 & 0.361 & 5.719 \\
\hline Parent Internalizing (T2) & Interaction (Adolescent Internalizing and Parent Coping) & 0.13 & 0.09 & 0.09 & 0.274 & -0.106 & 0.373 \\
\hline Parent Internalizing (T2) & Adolescent Sex & -0.42 & -0.04 & 0.05 & 0.459 & -1.555 & 0.702 \\
\hline Parent Internalizing (T2) & Adolescent Mean Age & 0.59 & 0.07 & 0.04 & 0.143 & -0.201 & 1.389 \\
\hline Parent Internalizing (T2) & Maternal Ethnicity & -0.53 & -0.04 & 0.05 & 0.382 & -1.730 & 0.663 \\
\hline Parent Internalizing (T2) & Time Between Stay-at-Home Order (Parent) & 0.07 & 0.04 & 0.05 & 0.470 & -0.086 & 0.251 \\
\hline Parent Internalizing (T2) & Time Between Assessments (Parent) & -0.01 & -0.01 & 0.06 & 0.804 & -0.110 & 0.100 \\
\hline
\end{tabular}

Adolescent sex is coded as $1=$ Male and $0=$ Female; Maternal Ethnicity is coded as Non-Hispanic, White $=1$ and Other $=0$; T1 and T2 represent the first and second assessments for adolescent and parent participants during the COVID-19 pandemic, respectively

Bold indicates $p<0.05$

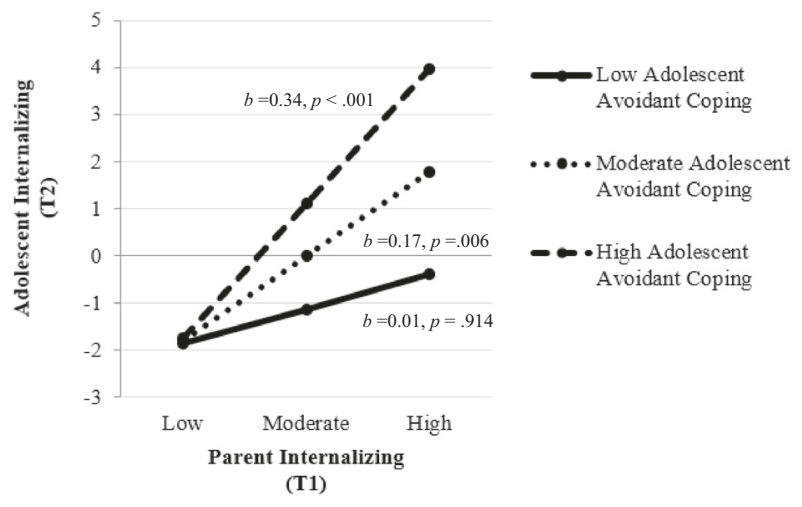

Fig. 2 Simple slopes for the interaction between $\mathrm{T} 1$ parent internalizing predicting $\mathrm{T} 2$ adolescent internalizing at different levels of $\mathrm{T} 1$ adolescent avoidant coping. Low adolescent avoidant coping was defined as $-1 \mathrm{SD}$ and high adolescent avoidant coping as $+1 \mathrm{SD}$ from the mean

\section{Cross-Lagged Moderation Model of Adolescent and Parent Anxiety Symptoms and Avoidant Coping}

The cross-lagged moderation model fit the data well, $\mathrm{RMSEA}=0.01 \quad[90 \% \quad \mathrm{CI}=0.00-0.04] ; \quad \mathrm{CFI}=0.99$; $\mathrm{SRMR}=0.04$. Parent and adolescent anxiety symptoms showed moderate to high stability from $\mathrm{T} 1$ to $\mathrm{T} 2$. However, there were no cross-lagged effects, direct effects of avoidant coping, or moderation effects.

\section{Cross-Lagged Moderation Model of Adolescent and Parent Depressive Symptoms and Avoidant Coping}

The cross-lagged moderation model results for depressive symptoms were similar to the internalizing symptoms model. Therefore, to account for the high correlation between anxiety and depressive symptoms, T2 parent and adolescent anxiety symptoms were also controlled for in the model. The model fit the data well, RMSEA $=0.02[90 \%$ $\mathrm{CI}=0.00-0.05] ; \mathrm{CFI}=0.99 ; \mathrm{SRMR}=0.04$. All unstandardized and standardized path coefficients, including the covariates, are reported in Table 3. Parent and adolescent depressive symptoms showed moderate to high stability from $\mathrm{T} 1$ to $\mathrm{T} 2$. There was also a direct effect of T1 adolescent avoidant coping on $\mathrm{T} 2$ adolescent depressive symptoms. There were no direct cross-lagged effects between adolescent and parent depressive symptoms between $\mathrm{T} 1$ and $\mathrm{T} 2$.

However, when examining the moderating effect of coping, the results showed that the relation between $\mathrm{T} 1$ parent depressive symptoms and $\mathrm{T} 2$ adolescent depressive symptoms was moderated by $\mathrm{T} 1$ adolescent avoidant coping, $b=0.29, S E=0.13, p=0.023$. As shown in Fig. 3, higher $\mathrm{T} 1$ parent depressive symptoms predicted higher T2 adolescent depressive symptoms when adolescent avoidant coping was high (i.e., $+1 \mathrm{SD}), \quad b=0.29 S E=0.10$, 


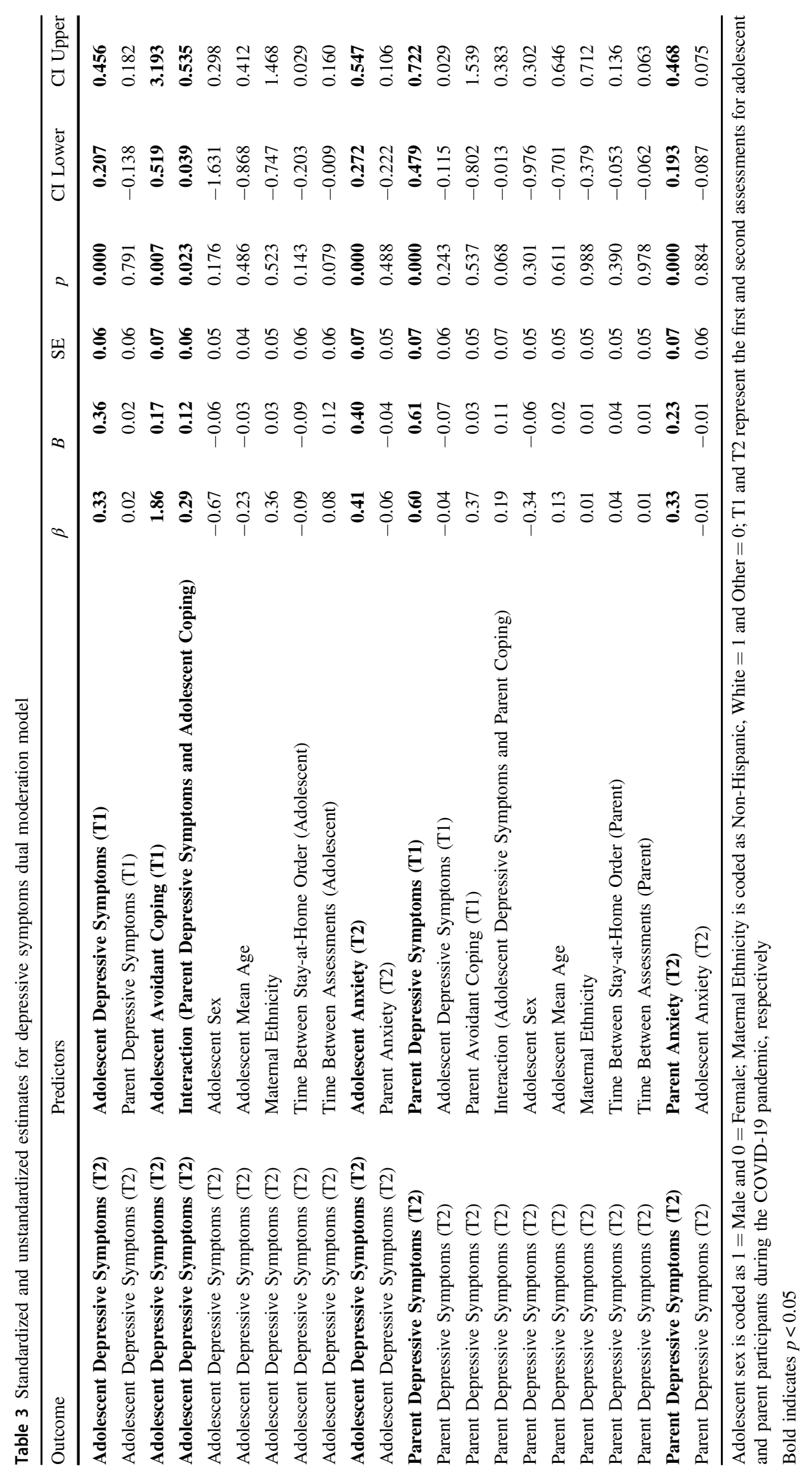




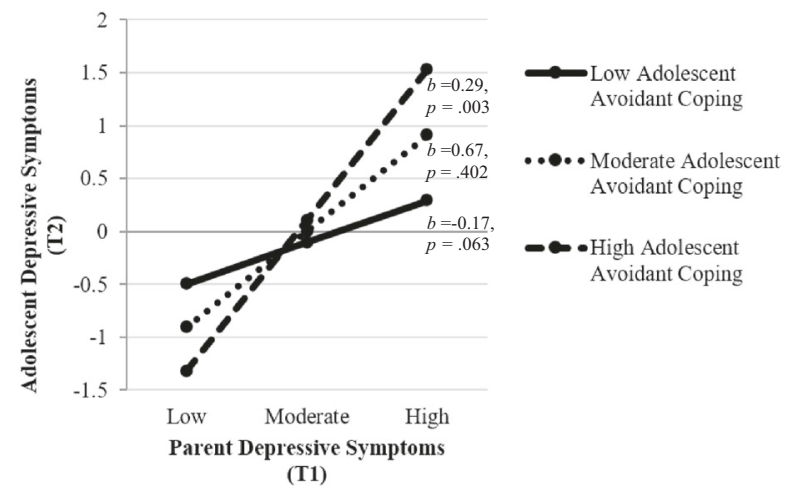

Fig. 3 Simple slopes for the interaction between $\mathrm{T} 1$ parent depressive symptoms predicting $\mathrm{T} 2$ adolescent depressive symptoms at different levels of T1 adolescent avoidant coping. Low adolescent avoidant coping was defined as $-1 \mathrm{SD}$ and high adolescent avoidant coping as $+1 \mathrm{SD}$ from the mean

$p=0.003$, but not when adolescent avoidant coping was moderate (i.e., mean level) $b=0.06, S E=0.07, p=0.402$, or low (i.e., $-1 \mathrm{SD}$ ), $b=-0.18, S E=0.07, p=0.063$. There was no significant interaction between $\mathrm{T} 1$ adolescent depressive symptoms and $\mathrm{T} 1$ parent avoidant coping on $\mathrm{T} 2$ parent depressive symptoms, $b=0.19, S E=0.10, p=$ 0.068 . These results revealed a similar pattern even when not accounting for anxiety in the model.

\section{Discussion}

The COVID-19 pandemic was an unprecedented life event that has the potential to negatively impact parent and adolescent internalizing symptoms in both the short- and long-term. The COVID-19 pandemic resulted in many US states and countries around the world enacting quarantines and stay-at-home orders. Although adolescence is a developmental period associated with increased independence, these changes resulted in many adolescents attending school virtually, missing out on significant social interactions, and spending considerably more time at home with their families (Lee, 2020). These changes have been found in cross-sectional studies to increase both parent and adolescent anxiety and depressive symptoms (Racine et al., 2020). Given research demonstrating that parents and children impact each other's internalizing symptoms in a transactional manner (Morris et al. 2007), it is important to examine the bidirectional relations between parent and adolescent depressive and anxiety symptoms, during the first few months of the COVID-19 pandemic in order to better understand the impact of each on the other during an unprecedented life event. The current longitudinal study aimed to address this by examining the cross-lagged effects between parent and adolescent internalizing symptoms (i.e., anxiety and depressive symptoms) during the first two months following stay-at-home orders in the state of Maryland during the COVID-19 pandemic. Furthermore, individuals who engage in high levels of avoidant coping may be affected by others' internalizing symptoms to a greater extent due to not effectively coping with the negative emotions they elicit, particularly during an unprecedented life event. As such, the extent to which avoidant coping moderated relations between parent and adolescent internalizing symptoms was examined.

Using cross-lagged analysis of parent and adolescent internalizing symptoms and coping, the current study found higher levels of parent internalizing symptoms, during the first month after the stay-at-home orders of the COVID 19 pandemic, predicted higher levels of adolescent internalizing symptoms one month later, especially for adolescent participants who engaged in avoidant coping behaviors. The findings extend previous results showing that parent internalizing symptoms can impact child internalizing symptoms over time, even through late adolescence (Ohannessian et al. 2005), by demonstrating this relation during the unprecedented global COVID-19 pandemic. This may be the case particularly given the circumstances of the COVID-19 pandemic, which resulted in many adolescents spending increased time with parents and decreased time with peers.

As hypothesized, the current study found that parent internalizing problems predicted the internalizing problems of late adolescents who engaged in moderate and high rates of avoidant coping. This is likely due to difficulty coping with an additional stressor (i.e., parent internalizing symptoms), especially during a particularly difficult time (i.e., COVID-19 pandemic). Late adolescents who engage in avoidant coping behaviors may not be effectively coping with the negative feelings emerging as a result of witnessing their parents' internalizing problems, and therefore they are more likely to internalize their negative feelings.

Contrary to what was expected, parent avoidant coping did not moderate the relation between adolescent internalizing symptoms during the first month post stay-at-home orders and parent internalizing symptoms one month later. Lower endorsement and variability of parent avoidant coping behaviors may have contributed to these null findings. Additionally, it is important to note the stability of internalizing symptoms for adolescent participants, and in particular for parent participants, which may have accounted for the non-significant moderation effects.

Sensitivity analyses examining depressive and anxiety symptoms in separate models suggest the effect of parents on adolescents may be particularly true with regard to depressive symptoms. Given that the same patterns were not demonstrated when only examining anxiety symptoms, it may be that parent depressive symptoms combined with 
adolescent avoidant coping is particularly harmful for adolescents' depressive symptoms. However, it is important to note that direct effects of parent depressive symptoms on adolescent depressive symptoms were not significant unlike the significant effect of $\mathrm{T} 1$ parent internalizing symptoms on $\mathrm{T} 2$ adolescent internalizing symptoms. One possible explanation for this null finding is that the combination of anxiety and depressive symptoms, or the increased variability in symptoms when combined, impact adolescent symptoms. Given the sample size, the current study was not powered to analyze both anxiety and depressive within one model as separate constructs. Future research should examine these constructs simultaneously with a larger sample to further tease apart these effects.

These findings suggest that parent internalizing symptoms do not impact all adolescents' internalizing symptoms. Additionally, these findings highlight the importance of targeting parent internalizing symptoms which may ultimately improve parent and adolescent internalizing symptoms. Furthermore, targeting a change in coping skills from avoidant to more active and positive coping may act as a buffer against other risk factors such as parental internalizing symptoms. For example, engaging in positive reappraisal has been demonstrated to be an active coping strategy (Garland et al., 2009) that increases positive affect and decreases negative affect in response to a stressful event (Rood et al., 2012). On the other hand, it may be that parents with higher rates of internalizing symptoms are less involved or engaged with their child, suggesting the need to target parenting and emotion coaching.

It is important to note that parents and adolescents reported on their anxiety and depressive symptoms approximately a month after stay-at-home orders were implemented in Maryland, where the majority of the participants resided. In the United States, prior to the pandemic, prevalence rates were approximately $3 \%$ for generalized anxiety in adolescents and adults (Merikangas et al., 2010) and $7 \%$ and $13 \%$ for depression in adults and adolescents, respectively (Center for Behavioral Health Statistics, 2018). The current study suggested higher rates of generalized anxiety symptoms in adolescents (i.e., $21 \%$ and $18 \%$ ) and adults (i.e., $8 \%$ and 6\%), and depressive symptoms in adolescents (i.e., 30\% and 25\%) and adults (i.e., 9\% and $8 \%$ ), at $\mathrm{T} 1$ and $\mathrm{T} 2$, respectively. These findings are similar to rates reported from other countries including China (Shi et al. 2020), Italy, and Spain during the COVID-19 pandemic (Orgiles et al. 2020). Notably, rates of both anxiety and depressive symptoms decreased across the two assessments. This may be a result of the gradual reopening which may have provided some sense of relief for families adapting to a "new normal," but further examination of internalizing symptoms throughout the COVID pandemic is warranted.
The current study has several notable strengths including two assessments of anxiety and depressive symptoms during the first few months of the COVID-19 pandemic. However, it is important to note that there was not an assessment right before the start of the COVID-19 pandemic to examine changes as a direct result of the COVID-19 pandemic. Furthermore, the current study sample was composed of predominantly well-educated, White families mostly living in the Mid-eastern United States, and therefore should not be generalized to other populations that may be disproportionately impacted negatively by the COVID-19 pandemic (Novacek et al. 2020). Recent reports have demonstrated significant disparities on the impact of the COVID-19 pandemic, including getting the virus (Karaca-Mandic et al. 2020), which the current sample was fairly protected from, given their background and education levels. Additionally, although the average age of participants was 18 years old $(\mathrm{SD} .=0.665)$ and adolescent age was accounted for in all analyses, it is important to note that a few participants had just turned 19 and a few were 16 which may represent differences in how they experienced the restrictions associated with the COVID-19 pandemic. Furthermore, the reliability of the parent avoidant coping scale was questionable. This may be a result of limited variability in responses endorsed by parents, which may have limited the possibility of significant findings in the current sample. Lastly, the current study focused on internalizing symptoms and did not examine the effects of the COVID-19 pandemic on externalizing symptoms (i.e., substance use, aggression).

\section{Conclusion}

Given the high rates of anxiety and depressive symptoms during the COVID-19 pandemic, it is important to understand how parents and adolescents are impacting each other in a time where restrictions have resulted in limited social interactions and increased family contact. The current study demonstrates the impact of parent internalizing symptoms on their late adolescent's internalizing symptoms longitudinally during a global pandemic. This is particularly true for adolescents who also engage in moderate and high levels of avoidant coping (e.g., blaming others, using substances). Findings emphasize the importance of targeting coping skills to address the impact of parent internalizing symptoms and other life stressors, such as the COVID-19 pandemic on late adolescent internalizing symptoms. Furthermore, findings highlight that parental anxiety and depressive symptoms do not only affect children when they are young but extends these findings to late adolescence. These findings can help us begin to pinpoint families most at-risk 
for internalizing symptoms during an unprecedented life event, such as the COVID-19 pandemic.

Acknowledgements We would like to acknowledge and thank the research assistants who helped collect the data for this study. Thank you Abby Brustad, Collin Drexler, and Christine Holm.

Authors' Contributions N.L. conceived of the study, participated in its design and coordination, interpretation of the data, performed the statistical analyses and drafted the manuscript; S.Z. participated in the design and interpretation of the data; S.M. participated in the design and interpretation of the data; J.L. helped to draft the manuscript and coordination of the study; K.D. helped to draft the manuscript; A.A. helped to draft the manuscript; H.H. helped to draft the manuscript; A. C.T. participated in the interpretation of the data and helped to draft the manuscript; N.F. conceived of the study and helped to draft the manuscript. All authors read and approved the final version of the manuscript.

Funding This work was supported by the National Institute of Mental Health (U01MH093349) and (R01MH118320 - 02S1).

\section{Compliance with Ethical Standards}

Conflict of Interest The authors declare that they have no conflict of interest.

Ethical Approval The study was performed in accordance with ethical standards. The Institutional Review Board at the University of Maryland approved all study protocols meeting ethical guidelines.

Informed Consent Informed consent was obtained from all individual participants included in the study.

Publisher's note Springer Nature remains neutral with regard to jurisdictional claims in published maps and institutional affiliations.

\section{References}

Baumstarck, K., Alessandrini, M., Hamidou, Z., Auquier, P., Leroy, T., \& Boyer, L. (2017). Assessment of coping: a new french four-factor structure of the brief COPE inventory. Health and Quality of Life Outcomes, 15, 1-9. https://doi.org/10.1186/s12955-016-0581-9.

Cameron, E. E., Joyce, K. M., Delaquis, C. P., Reynolds, K., Protudjer, J. L. P., \& Roos, L. E. (2020). Maternal psychological distress \& mental health service use during the COVID-19 pandemic. Journal of Affective Disorders, 276, 765-774. https://doi. org/10.1016/j.jad.2020.07.081.

Carver, C. S. (1997). You want to measure coping but your protocol's too long: Consider the brief COPE. International Journal of Behavioral Medicine, 4, 92-100. https://doi.org/10.1207/ s15327558ijbm0401_6.

Center for Behavioral Health Statistics. (2018). 2017 National Survey on Drug Use and Health: Methodological Summary and Definitions.

Ciciolla, L., Gerstein, E. D., \& Crnic, K. A. (2014). Reciprocity Among Maternal Distress, Child Behavior, and Parenting: Transactional Processes and Early Childhood Risk. Journal of Clinical Child \& Adolescent Psychology, 43, 751-764. https:// doi.org/10.1080/15374416.2013.812038.

Ehring, T., Razik, S., \& Emmelkamp, P. M. G. (2011). Prevalence and predictors of posttraumatic stress disorder, anxiety, depression, and burnout in Pakistani earthquake recovery workers. Psychiatry Research, 185, 161-166. https://doi.org/10.1016/j.psychres.2009. 10.018.

Enders, C. K., \& Bandalos, D. L. (2001). The relative performance of full information maximum likelihood estimation for missing data in structural equation models. Structural Equation Modeling, 8 (3), 430-457. https://doi.org/10.1207/S15328007SEM0803_5.

Garland, E., Gaylord, S., \& Park, J. (2009). The Role of Mindfulness in Positive Reappraisal. Explore, 5, 37-44. https://doi.org/10. 1016/j.explore.2008.10.001.

Goodman, S. H., Rouse, M. H., Connell, A. M., Broth, M. R., Hall, C. M., \& Heyward, D. (2011). Maternal Depression and Child Psychopathology: A Meta-Analytic Review. Clinical Child and Family Psychology Review, 14, 1-27. https://doi.org/10. 1007/s10567-010-0080-1.

Hane, A. A., Fox, N. A., Henderson, H. A., \& Marshall, P. J. (2008). Behavioral reactivity and approach-withdrawal bias in infancy. Developmental Psychology, 44(5), 1491-1496. https://doi.org/10. 1037/a0012855.

Karaca-Mandic, P., Georgiou, A., \& Sen, S. (2020). Assessment of COVID-19 Hospitalizations by Race/Ethnicity in 12 States. JAMA Internal Medicine, 19-21. https://doi.org/10.1001/jama internmed.2020.3857.

Kerns, C. E., Elkins, R. M., Carpenter, A. L., Chou, T., Green, J. G., \& Comer, J. S. (2014). Caregiver distress, shared traumatic exposure, and child adjustment among area youth following the 2013 Boston Marathon bombing. Journal of Affective Disorders, 167, 50-55. https://doi.org/10.1016/j.jad.2014.05.040.

Kroenke, K., Baye, F., \& Lourens, S. G. (2019). Comparative validity and responsiveness of PHQ-ADS and other composite anxietydepression measures. Journal of Affective Disorders, 246, 437-443. https://doi.org/10.1016/j.jad.2018.12.098.

Kroenke, K., Strine, T. W., Spitzer, R. L., Williams, J. B. W., Berry, J. T., \& Mokdad, A. H. (2009). The PHQ-8 as a measure of current depression in the general population. Journal of Affective Disorders, 114(1-3), 163-173. https://doi.org/10.1016/j.jad.2008.06.026.

Kroenke, K., Wu, J., Yu, Z., Bair, M. J., Kean, J., Stump, T., Monahan, P. O., \& Author, P. M. (2016). The Patient Health Questionnaire Anxiety and Depression Scale (PHQ-ADS): Initial Validation in Three Clinical Trials HHS Public Access Author manuscript. Psychosom Med, 78, 716-727. https://doi.org/10. 1097/PSY.0000000000000322.

Lee, J. (2020). Mental health effects of school closures during COVID-19. The Lancet Child and Adolescent Health, 4, 421 https://doi.org/10.1016/S2352-4642(20)30109-7.

Merikangas, K. R., He, J., Burstein, M., Swanson, S. A., Avenevoli, S., Cui, L., Benjet, C., Georgiades, K., \& Swendsen, J. (2010). Lifetime Prevalence of Mental Disorders in U.S. Adolescents: Results from the National Comorbidity Survey Replication-Adolescent Supplement (NCS-A). Journal of the American Academy of Child \& Adolescent Psychiatry, 49, 980-989. https://doi.org/10.1016/j.jaac. 2010.05.017.

Morris, A. S., Silk, J. S., Steinberg, L., Myers, S. S., \& Robinson, L. R. (2007). The role of the family context in the development of emotion regulation. Social Development, 16(2), 361-388. https:// doi.org/10.1111/j.1467-9507.2007.00389.x.

Muthén, L.K. and Muthén, B.O. (1998-2017). Mplus User's Guide. Eighth Edition. Los Angeles, CA: Muthén \& Muthén

Novacek, D. M., Hampton-Anderson, J. N., Ebor, M. T., Loeb, T. B., \& Wyatt, G. E. (2020). Mental Health Ramifications of the COVID-19 Pandemic for Black Americans: Clinical and Research Recommendations. Psychological Trauma: Theory. Research, Practice, and Policy, 12, 449-451. https://doi.org/10.1037/tra0000796.

Ohannessian, C. M. C., Hesselbrock, V. M., Kramer, J., Kuperman, S., Bucholz, K. K., Schuckit, M. A., \& Nurnberger, J. I. (2005). The relationship between parental psychopathology and adolescent 
psychopathology: An examination of gender patterns. Journal of Emotional and Behavioral Disorders, 13, 67-76. https://doi.org/ 10.1177/10634266050130020101.

Ollendick, T. H., Langley, A. K., Jones, R. T., \& Kephart, C. (2001). Fear in Children and Adolescents: Relations with Negative Life Events, Attributional Style, and Avoidant Coping. Journal of Child Psychology and Psychiatry, 42, 1029-1034. https://doi.org/ 10.1111/1469-7610.00801.

Orgiles, M., Morales, A., Delveccio, E., Mazzeschi, C., Espada, J. P. (2020). Immediate Psychological Effects of COVID-19 Quarantine in Youth from Italy and Spain. Available at SSRN: https://ssrn. com/abstract $=3588552$ or https://doi.org/10.2139/ssrn.3588552.

Pahayahay, A., \& Khalili-Mahani, N. (2020). What media helps, what media hurts: a mixed methods survey study of coping with COVID-19 using the media repertoire framework and the appraisal theory of stress. Journal of Medical Internet Research, 22, e20186 https://doi.org/10.2196/20186.

Park, A., Velez, C. V., Kannan, K., \& Chorpita, B. F. (2020). Stress, functioning, and coping during the COVID-19 pandemic: Results from anonline convenience sample. https://doi.org/10.31234/osf.io/ jmctv.

Pereira, A. I., Barros, L., Mendonça, D., \& Muris, P. (2014). The Relationships Among Parental Anxiety, Parenting, and Children's Anxiety: TheMediating Effects of Children's Cognitive Vulnerabilities. Journal of Child and Family Studies, 23, 399-409. https://doi.org/10.1007/s10826-013-9767-5.

Racine, N., Cooke, J. E., Eirich, R., Korczak, D. J., McArthur, B., \& Madigan, S. (2020). Child and adolescent mental illness during COVID-19: A rapid review. Psychiatry Research, 292, 113307 https://doi.org/10.1016/j.psychres.2020.113307.

Rood, L., Roelofs, J., Bögels, S. M., \& Arntz, A. (2012). The Effects of Experimentally Induced Rumination, Positive Reappraisal, Acceptance, and Distancing When Thinking About a Stressful Event on Affect States in Adolescents. Journal of Abnormal Child Psychology, 40, 73-84. https://doi.org/10.1007/s10802011-9544-0.

Seiffge-Krenke, I., \& Klessinger, N. (2000). Long-term effects of avoidant coping on adolescents' depressive symptoms. Journal of Youth and Adolescence, 29, 617-630. https://doi.org/10.1023/A: 1026440304695.

Shi, L., Lu, Z.-A., Que, J.-Y., Huang, X.-L., Liu, L., Ran, M.-S., Gong, Y.-M., Yuan, K., Yan, W., Sun, Y.-K., Shi, J., Bao, Y.-P., \& Lu, L. (2020). Prevalence of and risk factors associated with mental health symptoms among the general population in china during the coronavirus disease 2019 pandemic. JAMA Network Open, 3(7), e2014053 https://doi.org/10.1001/jamanetworkopen.2020.14053.

Spitzer, R. L., Kroenke, K., Williams, J. B. W., \& Löwe, B. (2006). A brief measure for assessing generalized anxiety disorder: The GAD-7. Archives of Internal Medicine, 166, 1092-1097. https:// doi.org/10.1001/archinte.166.10.1092.

Wilkinson, R. B., Walford, W. A., \& Espnes, G. A. (2000). Coping styles and psychological health in adolescents and young adults: A comparison of moderator and main effects models. Australian Journal of Psychology, 52, 155-162. https://doi.org/10.1080/ 00049530008255383.

Yusoff, M. S. B.(2011). The Validity of the Malay Brief Cope in Identifying. International Medical Journal, 18, 29-33.
Nicole E. Lorenzo is a Postdoctoral Fellow at the University of Maryland. Her major research interests include early intervention and the relation between parenting (including parenting practices and parental psychopathology) on early childhood social-emotional and behavioral problems.

Selin Zeytinoglu is a Postdoctoral Fellow at the University of Maryland. Her major research interests include the development of self-regulation (i.e., regulation of emotions, cognition, \& behaviors) over the course of childhood.

Santiago Morales is a Postdoctoral Fellow at the University of Maryland. His major research interests include the development of emotion, emotion regulation, and temperament in children by using psychophysiological, neurobiological, and behavioral measures.

Jamie Listokin is a project coordinator at the University of Maryland. Her major research interests include how early experiences shape brain development and, in turn, individuals' adaptive and maladaptive outcomes.

Alisa N. Almas is a Faculty Research Associate at the University of British Columbia. Her major research interests include social development across childhood and adolescence, with a special interest in children in at-risk or atypical populations.

Kathryn A. Degnan is an Assistant Professor at the Catholic University. Her major research interests include identifying and defining early individual differences in emotion and behavior (i.e., temperament), with a particular focus on the transactional processes between individuals and the surrounding social context (e.g., parenting, peers) that contributes to risk and resilience throughout childhood, adolescence, and adulthood.

Heather Henderson is a Professor at Waterloo University. Her major research interests include (a) the role of early temperament on children's social development, (b) affective and cognitive influences on self-regulation in typically- and atypically-developing children, and (c) developmental psychophysiology.

Andrea Chronis-Tuscano is a Professor at the University of Maryland. Her major research interests include understanding early predictors of developmental outcomes for children with AttentionDeficit/Hyperactivity Disorder (including depression and alcohol/ substance use) and developing novel treatments which target these early risk and protective factors. parental psychopathology, parenting, and early intervention.

Nathan A. Fox is a Distinguished University Professor at the University of Maryland. His major research interests include infant and child temperament, development of emotion and emotion regulation, human developmental neuroscience, and infant social cognition. 